01,07

\title{
Анализ скоростных зависимостей критических напряжений в алюминиевых сплавах системы Al-Mg при ударных нагрузках
}

\author{
() А.Д. Евстифеев ${ }^{1}$, И.В. Смирнов ${ }^{2}$, Ю.В. Петров ${ }^{2,3}$ \\ ${ }^{1}$ Научно-исследовательский институт механики Национального исследовательского \\ Нижегородского государственного университета им. Н.И. Лобачевского, \\ Нижний Новгород, Россия \\ ${ }^{2}$ Санкт-Петербургский государственный университет, \\ Санкт-Петербург, Россия \\ ${ }^{3}$ Институт проблем машиноведения РАН, \\ Санкт-Петербург, Россия \\ E-mail: ad.evstifeev@gmail.com
}

Поступила в Редакцию 2 апреля 2020 г.

В окончательной редакции 21 июня 2020 г.

Принята к публикации 23 июня 2020 г.

Алюминиевые сплавы системы $\mathrm{Al}-\mathrm{Mg}$ имеют широкое распространение в автомобильной и авиационной промышленности благодаря сочетанию прочности, хорошей пластичности и коррозионной стойкости. Увеличение процентного содержания магния повышает прочность материала при незначительном снижении пластических характеристик. В работе изучены особенности поведения критических напряжений в рассматриваемых материалах, наблюдаемые при увеличении скорости деформации. Обсуждается возможность прогнозирования критических динамических напряжений в материалах на основе структурно-временного подхода.

Ключевые слова: алюминиевые сплавы, критические динамические напряжения, критерий инкубационного времени.

DOI: $10.21883 /$ FTT.2020.11.50048.077

\section{1. Введение}

Широкое применение материалов на основе алюминия требует всестороннего изучения прочностных характеристик материала в широком диапазоне изменения параметров внешнего нагружения. Особое внимание стоит уделять области экстремальных условий эксплуатации, поскольку поведение материала при повышенных скоростях нагружения и импульсном воздействии кардинальным образом отличается от работы в квазистатических режимах. Влияние на прочность материала в условиях динамических нагрузок оказывают химический состав материала, его структурное состояние, а также внешние факторы, например, температура.

Алюминиевые сплавы системы $\mathrm{Al}-\mathrm{Mg}$ благодаря хорошим функциональным свойствам [1] широко используются как в статическом, так и в динамическом диапазоне изменения параметров внешнего воздействия. Увеличение процентного содержания магния повышает прочность в статическом диапазоне нагружения при незначительном снижении пластических характеристик. При одноосном растяжении предельная деформация до разрушения может достигать 30\%. При этом поведение в динамическом диапазоне недостаточно исследовано, несмотря на то, что с повышением скорости деформирования реакция материала существенно изменяется, например, повышается предельная прочность материала, связанная со структурно-временными особенностя- ми процесса разрушения [2-8]. Это требует экспериментального и теоретического изучения динамических прочностных свойств.

\section{2. Материалы и методика экспериментальных исследований}

\section{1. Материалы}

Наиболее широкое распространение в промышленности получили сплавы с содержанием магния от 1 до 5\%. Увеличение содержания магния свыше 6\% приводит к ухудшению коррозионной стойкости сплава. При этом в нагартованном состоянии механическая структура сплава становится нестабильной.

В настоящей работе рассматриваются сплавы системы Al-Mg: AMg2, AMg3, Al5083 (AMg4.5), AMg5, AMg6. Сплавы $\mathrm{AMg} 2$ и $\mathrm{AMg} 3$ в состоянии поставки были в виде листов толщиной $1.5 \mathrm{~mm}$. Сплав A15083 (AMg4.5) был поставлен в виде литой плиты без первоначальной деформационной обработки. Сплавы AMg5 и AMg6 были приобретены в виде экструдированных прутков диаметром $20 \mathrm{~mm}$. С целью исключения влияния предварительной деформации на результаты механических испытаний все материалы подвергались релаксационному отжигу при $633 \mathrm{~K}$.

Для улучшения прочностных характеристик сплавы системы $\mathrm{Al}-\mathrm{Mg}$ легируют хромом, марганцем, титаном, 
Таблица 1. Химический состав алюминиевых сплавов системы $\mathrm{Al}-\mathrm{Mg}$

\begin{tabular}{c|c|c|c|c|c}
\hline \multirow{2}{*}{ Сплав } & \multicolumn{5}{|c}{ Элемент, $(\mathrm{wt} \%)$} \\
\cline { 2 - 6 } & $\mathrm{Mg}$ & $\mathrm{Mn}$ & $\mathrm{Fe}$ & $\mathrm{Si}$ & $\mathrm{Al}$ \\
\hline $\mathrm{AMg} 2$ & 2.3 & 0.2 & 0.2 & 0.1 & Balance \\
$\mathrm{AMg} 3$ & 3.6 & 0.5 & 0.3 & 0.6 & Balance \\
$\mathrm{AMg} 4.5$ & 4.5 & 0.5 & 0.5 & 0.5 & Balance \\
(A15083) & 5.3 & 0.6 & 0.3 & 0.2 & Balance \\
$\mathrm{AMg} 5$ & 5.8 & 0.3 & 0.3 & 0.2 & Balance
\end{tabular}

кремнием или ванадием. Химический состав рассматриваемых сплавов приведен в табл. 1. Магний является основным легирующим элементом. Примеси меди и железа в сплавы этой системы нежелательны, поскольку они снижают их коррозионную стойкость и свариваемость. При этом видно, что железо присутствует во всех материалах. Максимальное его количество содержится в сплаве $\mathrm{AMg} 4.5$.

\section{2. Методики квазистатических и динамических испытаний на растяжение}

Экспериментальное исследование статической и динамической прочности материалов было выполнено на образцах с рабочей частью $5 \mathrm{~mm}$ на $2 \mathrm{~mm}$ в длину и ширину соответственно. Установка ShimadzuAG-50 kNX использовалась для растяжения в квазистатических режимах при скорости деформации $5 \cdot 10^{-4} \mathrm{~s}^{-1}$, а установка Instron CEAST 9350 - для реализации ударных растягивающих нагрузок при скорости деформации порядка $10^{2}-10^{3} \mathrm{~s}^{-1}$.

Выполненные ранее исследования $[9,10]$ с использованием башенного копра и специальных захватов подтвердили работоспособность используемой схемы.

Известно, что для многих материалов, при увеличении скорости деформации критическое напряжение разрушения растет [11-14]. Однако фиксация скорости деформации на малых образцах является сложной задачей и в данном исследовании более приемлемым является контроль изменения критических напряжений в зависимости от вариации скорости роста напряжений [9]. Скорость роста напряжений также отражает чувствительность материала к интенсивности воздействия.

\section{3. Результаты и обсуждение}

\section{1. Результаты механических испытаний}

На рис. 1 представлены диаграммы одноосного деформирования сплавов $\mathrm{AMg} 2, \mathrm{AMg} 3, \mathrm{Al} 5083$ (AMg4.5), $\mathrm{AMg} 5, \mathrm{AMg} 6$ при скорости деформации $5 \cdot 10^{-4} \mathrm{~s}^{-1}$. $\mathrm{C}$ увеличением процентного содержания $\mathrm{Mg}$ в сплаве

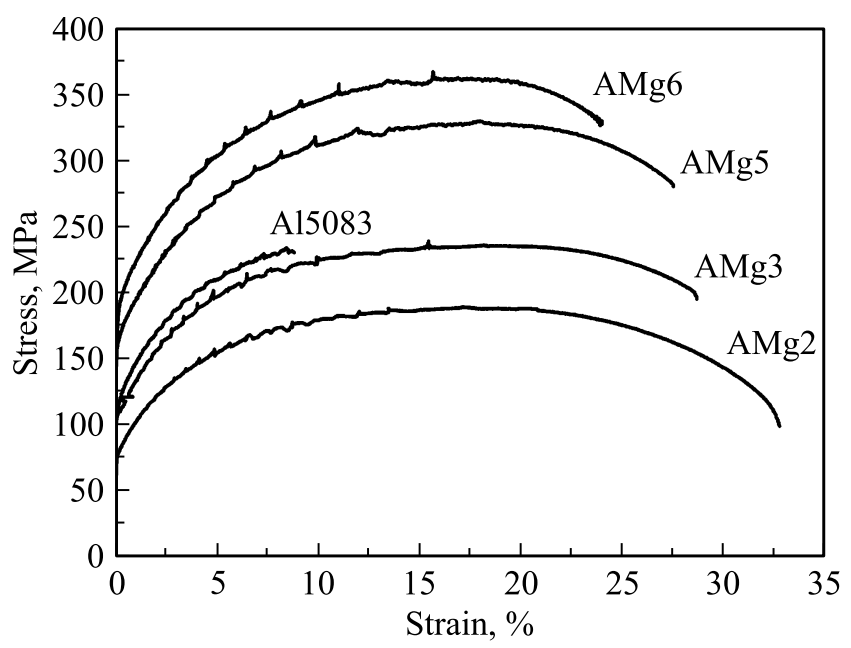

Рис. 1. Диаграммы одноосного деформирования сплавов $\mathrm{AMg} 2, \mathrm{AMg} 3, \mathrm{Al} 5083$ (AMg4.5), AMg5, AMg6 при скорости деформации $5 \cdot 10^{-4} \mathrm{~s}^{-1}$.

увеличивается прочность материала при незначительном снижении пластичности. На этом фоне выделяется сплав Al5083 (AMg4.5) производства фирмы Gleich Aluminium Gmbh. Материал поставляется в литом виде без сформированной текстуры $[15,16]$. Все остальные материалы подвергались первоначальной деформационной обработке.

Для оценки механической прочности были определены стандартные характеристики материалов (табл. 2): временное сопротивление разрыву $\sigma_{\mathrm{UTS}}$, условный предел текучести $\sigma_{0.2}$, относительное удлинение $\delta$.

Максимальное относительное удлинение образцов было зафиксировано для сплава $\mathrm{AMg} 2$, минимальное для $\mathrm{AMg} 4.5$, что соответствует их паспортным характеристикам. Также стоит отметить и снижение пластичности у сплава AMg6.

Среди полученных данных особый интерес представляют диаграммы сплавов $\mathrm{AMg} 3$ и $\mathrm{AMg} 4.5$. Данные сплавы имеют близкие значения прочности, но относительное удлинение различается в три раза. Это позволяет провести исследование динамической прочности материалов с близкими значениями прочности и различными характеристиками пластичности.

Таблица 2. Механические характеристики алюминиевых сплавов системы $\mathrm{Al}-\mathrm{Mg}$

\begin{tabular}{c|c|c|c|c}
\hline Сплав & $\begin{array}{c}\sigma_{0.2}, \\
\mathrm{MPa}\end{array}$ & $\begin{array}{c}\sigma_{\mathrm{UTS}}, \\
\mathrm{MPa}\end{array}$ & $\begin{array}{l}\delta, \\
\%\end{array}$ & $\begin{array}{c}\tau, \\
\mu \mathrm{s}\end{array}$ \\
\hline $\mathrm{AMg} 2$ & $71 \pm 0.5$ & $188 \pm 1$ & $33.5 \pm 0.5$ & 18 \\
$\mathrm{AMg3}$ & $105 \pm 1$ & $236 \pm 2$ & $28.6 \pm 0.7$ & 15 \\
$\mathrm{AMg} 4.5$ & $110 \pm 1$ & $235 \pm 2$ & $10 \pm 2$ & 16 \\
$(\mathrm{~A} 15083)$ & $156 \pm 2$ & $319 \pm 3$ & $29 \pm 1.5$ & 10 \\
$\mathrm{AMg5}$ & $170 \pm 2$ & $359 \pm 3$ & $23.8 \pm 1$ & 9 \\
$\mathrm{AMg6}$ & $170 \pm$
\end{tabular}




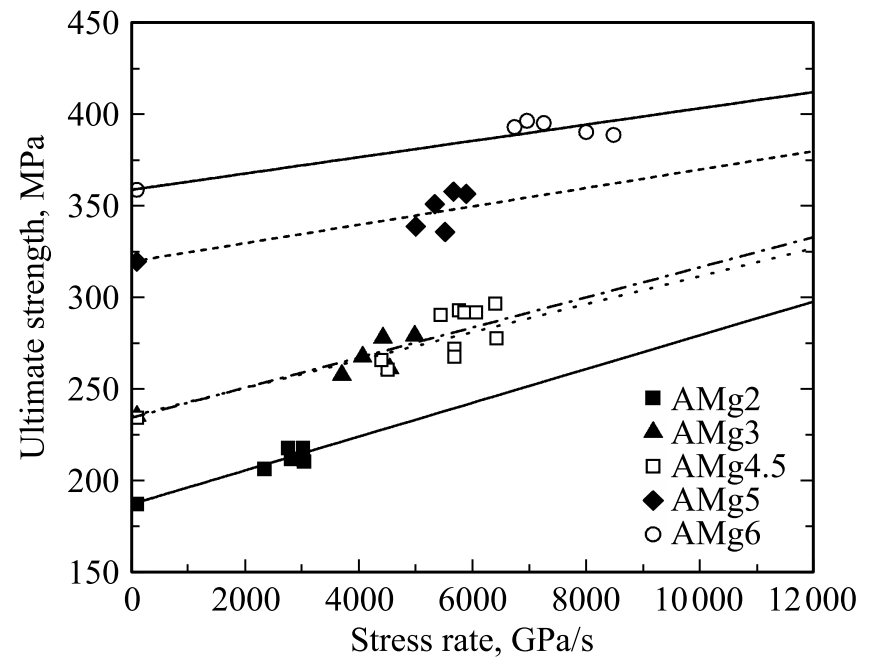

Рис. 2. Зависимость прочности на растяжение от скорости роста напряжений для алюминиевых сплавов системы $\mathrm{Al}-\mathrm{Mg}$. Динамические кривые построены по критерию (1) с параметрами материала (табл. 2).

Предельные величины прочности на растяжение для исследуемых материалов в зависимости от скорости роста напряжений представлены маркерами на рис. 2.
C ростом скорости приложения нагрузки для сплавов AMg5 и AMg6 коэффициент динамического упрочнения ниже чем у сплавов $\mathrm{AMg} 2, \mathrm{AMg} 3$ и $\mathrm{AMg} 4.5$.

Отмеченные особенности поведения материала при высоких скоростях нагружения можно объяснить с применением структурно-временного подхода. В качестве критерия разрушения материала при растяжении возьмем хорошо зарекомендовавший себя для динамических задач критерий инкубационного времени $[17,18]$ :

$$
\frac{1}{\tau} \int_{t-\tau}^{t} \frac{\sigma(s)}{\sigma_{\mathrm{UTS}}} d s \leq 1,
$$

где $t$ - время, $\sigma(s)$ - зависимость разрывающего напряжения от времени, $\sigma_{\mathrm{UTS}}-$ временное сопротивление разрыву при квазистатическом нагружении, $\tau$ - инкубационное время разрушения, являющееся мерой прочности в динамическом диапазоне параметров внешнего воздействия. Константы $\sigma_{\mathrm{UTS}}$ и $\tau$ являются параметрами прочности материала. Критерий позволяет объяснять и прогнозировать особенности скоростных зависимостей критических разрушающих напряжений в материалах, которые зачастую показывают нестабильное поведение, зависящее от параметров и способа приложения внешней нагрузки.
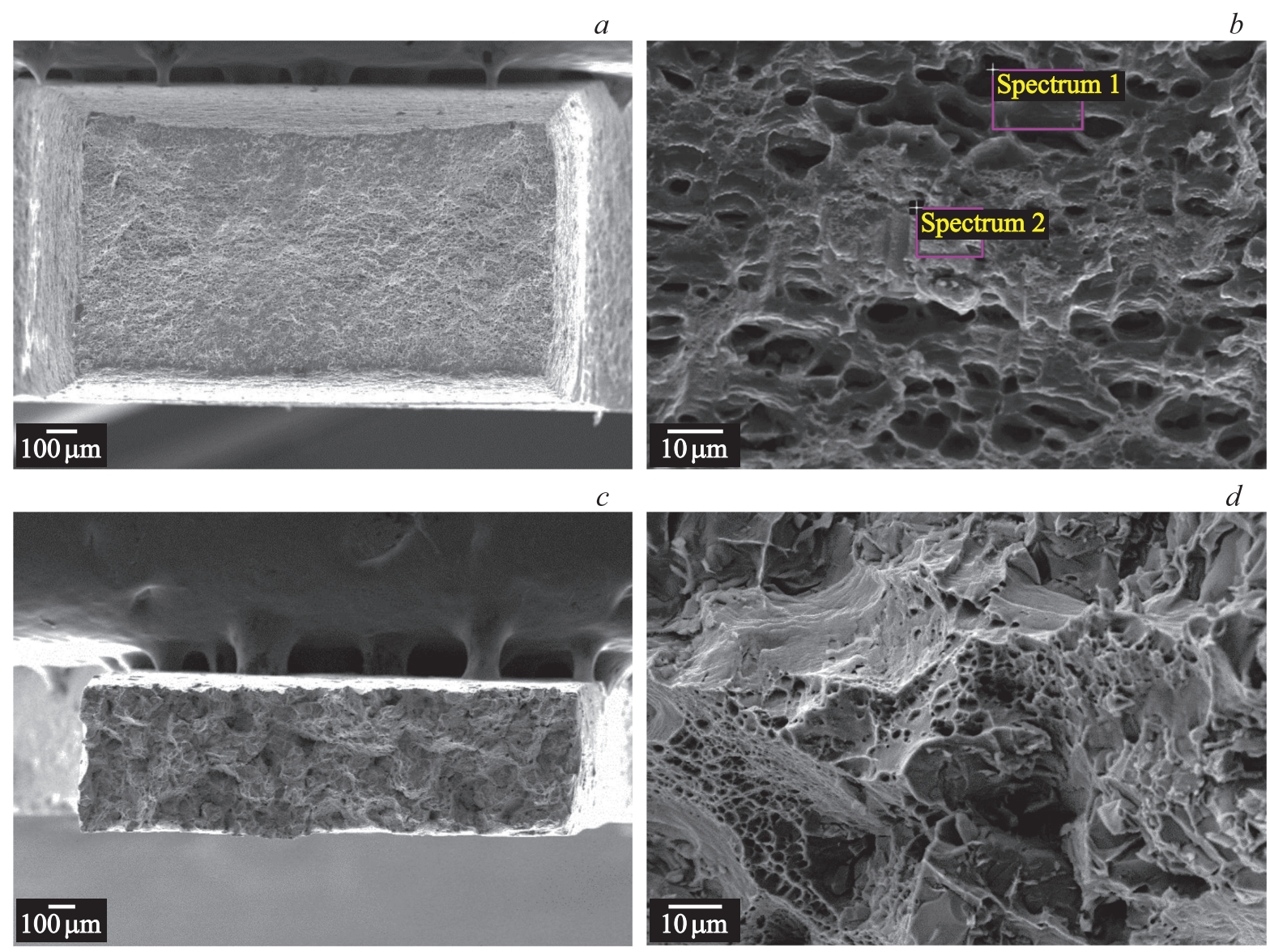

Рис. 3. Данные сканирующей электронной микроскопии. Фрактография образцов $a, b-\mathrm{AMg} 3 ; c, d-\mathrm{AMg} 4.5$ после растяжения при скорости деформации $5 \cdot 10^{-4} \mathrm{~s}^{-1}$. 

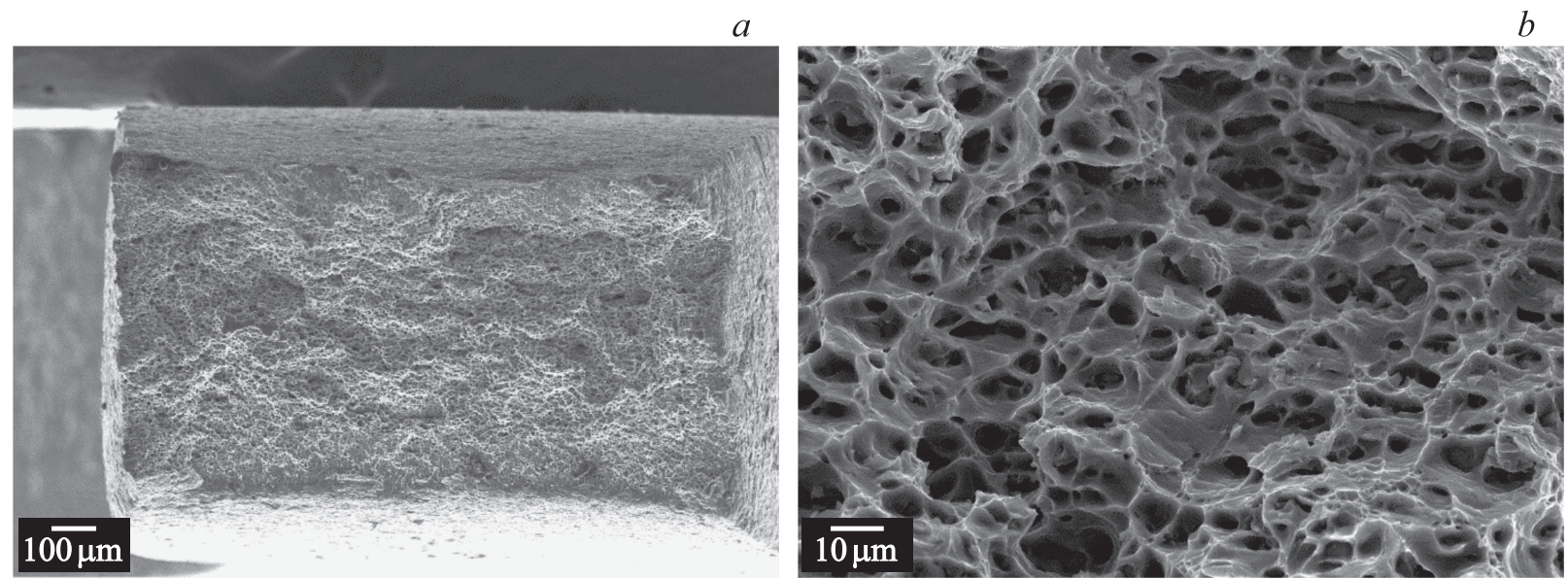

$c$
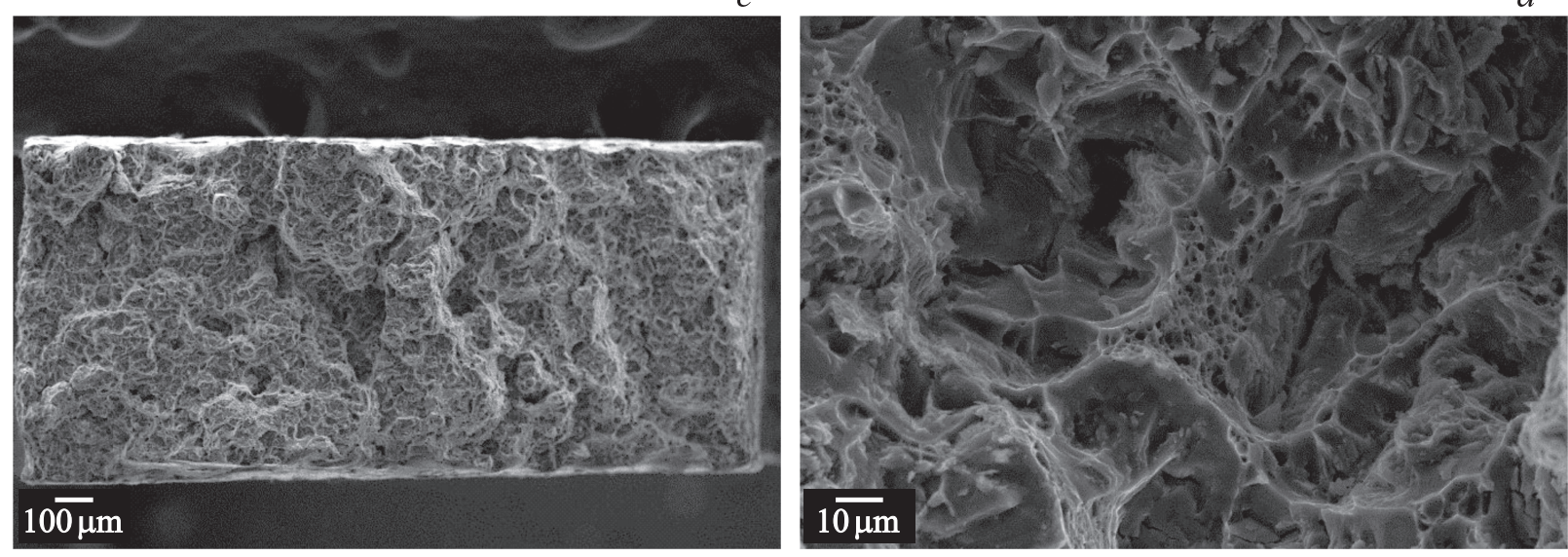

Рис. 4. Данные сканирующей электронной микроскопии. Фрактография образцов $a, b-\mathrm{AMg} 3 ; c, d-\mathrm{AMg} 4.5$ после растяжения при скорости $4400 \mathrm{GPa} / \mathrm{s}$.

В качестве модельного разрывающего напряжения был выбран импульс (2), линейно возрастающий во времени, что наиболее приближено к условиям ударного эксперимента

$$
\sigma(t)=\dot{\sigma} \cdot t \cdot H(t)
$$

где $H(t)$ - функция Хевисайда, $\dot{\sigma}-$ скорость роста напряжений. Подставляя выражение (2) в критерий (1) при достижении равенства находим момент времени разрушения как функцию от $\dot{\sigma}(3)$

$$
t^{*}=\left\{\begin{array}{ll}
\tau / 2+\sigma_{\mathrm{UTS}} / \dot{\sigma}, & t^{*} \geq \tau \\
\sqrt{2 \sigma_{\mathrm{UTS}} \cdot \tau / \dot{\sigma}}, & t^{*}<\tau
\end{array} .\right.
$$

Тогда из (2) следует следующее выражение для порогового напряжения

$$
\sigma^{*}=\sigma\left(t^{*}\right)= \begin{cases}\sigma_{\mathrm{UTS}}+\dot{\sigma} \cdot \tau / 2, & t^{*} \geq \tau \\ \sqrt{2 \dot{\sigma} \cdot \sigma_{\mathrm{UTS}} \cdot \tau}, & t^{*}<\tau\end{cases}
$$

Инкубационное время разрушения $\tau$ можно определить полуэмпирическим методом путем перебора величин параметра $\tau$ и сравнения получаемых величин с экспериментальными данными. Поиск искомой величины параметра заключается в минимизации отклонения теоретической кривой от экспериментальных точек. Итоговые величины $\tau$ для каждого из рассмотренных материалов приведены в табл. 2.

Анализируя полученные данные, представленные на рис. 2, можно отметить, что алюминиевые сплавы $\mathrm{AMg} 2, \mathrm{AMg} 3$ и $\mathrm{AMg} 4.5$ имеют примерно одинаковую динамическую прочность в терминах инкубационного времени. Это выражается в наклоне расчетной кривой в координатах предельное напряжение - скорость роста напряжений. С ростом скорости приложения нагрузки тенденция относительно соотношения прочностных характеристик, полученная в квазистатической области нагружения, сохраняется. В случае же сплавов AMg5 и AMg6 динамические кривые прочности имеют меньший наклон, что выражается меньшей динамической прочностью в терминах инкубационного времени. По всей видимости, полученные результаты связаны с различиями в химическом составе материалов. В случае динамического нагружения сплав AMg4.5 не выделяется из общей тенденции, несмотря на свои деформационные характеристики при квазистатическом нагружении. 

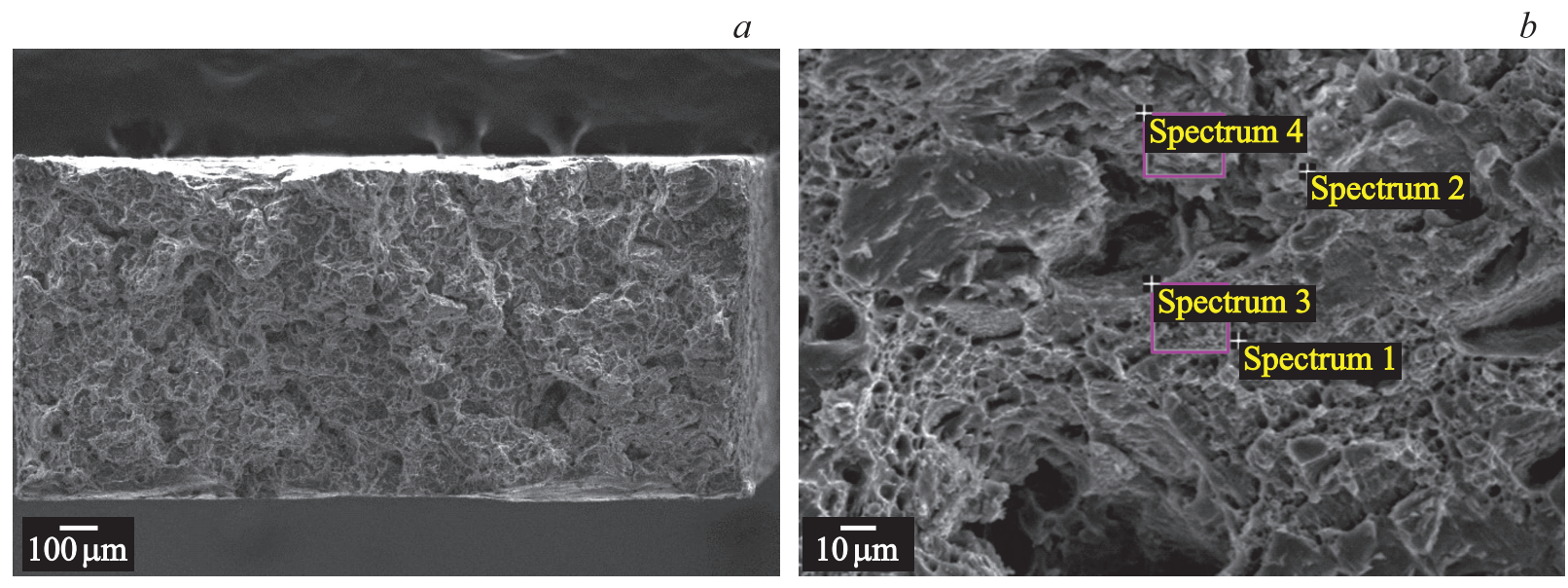

$c$
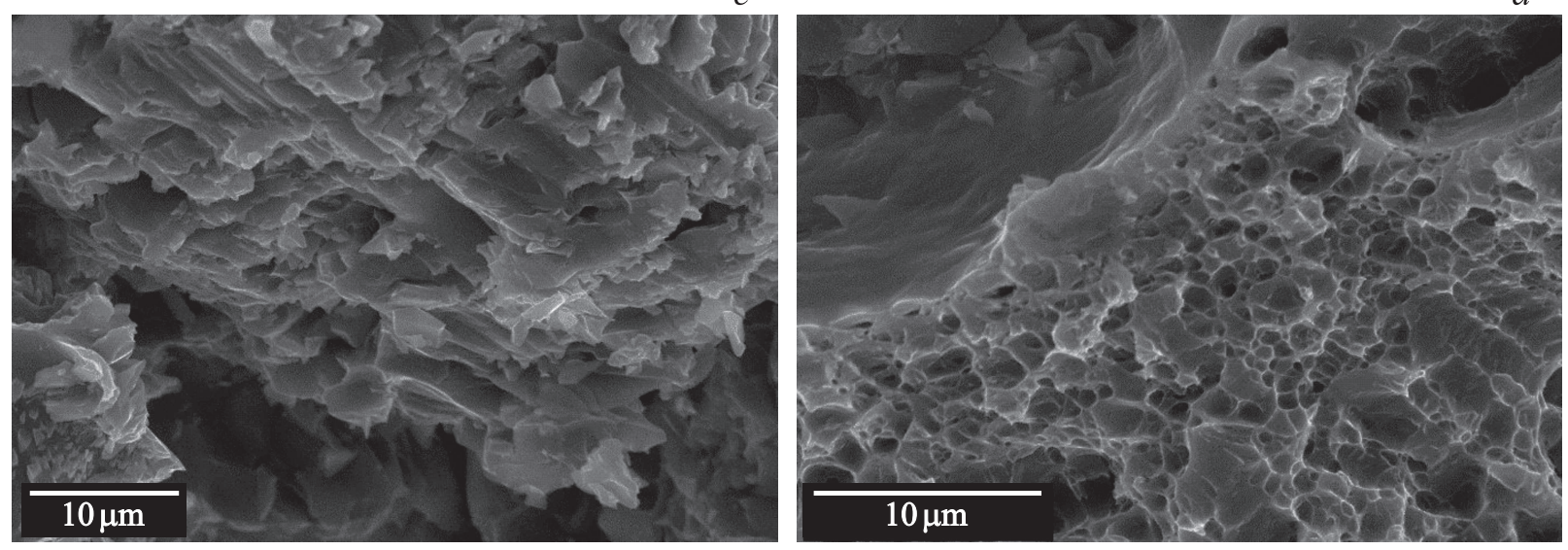

Рис. 5. Данные сканирующей электронной микроскопии. Фрактография образцов $\mathrm{AMg} 4.5$ после растяжения при скорости $5860 \mathrm{GPa} / \mathrm{s} ; c$ - область „Spectrum 4“; $d$ - область „Spectrum 3“.

Остановимся более детально на особенностях деформирования сплавов $\mathrm{AMg} 3$ и $\mathrm{AMg} 4.5$.

\section{2. Результаты анализа поверхности разрушения}

На рис. 3 представлены данные фрактографии поверхности разрушения образцов $\mathrm{AMg} 3$ и $\mathrm{AMg} 4.5$ после растяжения со скоростью $5 \cdot 10^{-4} \mathrm{~s}^{-1}$. Данные получены с использованием сканирующего электронного микроскопа Zeiss Merlin. Различия в деформационной картине кардинальные. Во-первых, для сплава $\mathrm{AMg} 3$ характерна локализация деформации, сопровождающаяся уменьшением сечения в среднем на 50\%, в то время как для сплава $\mathrm{AMg} 4.5$ только 17\%. Во-вторых, сплав $\mathrm{AMg} 3$ имеет более ровную поверхность разрушения, на которой выделяются две области с различными размерами ямок. В области с меньшими ямками (рис. 3, $b$ „Spectrum 1“) концентрируются $\mathrm{Si}, \mathrm{Mn}$ и $\mathrm{Fe}$. В области „Spectrum 2“ данные элементы отсутствуют. При этом, поскольку ямочная поверхность разрушения (рис. $3, b$, рис. $4, b$ ) не обнаруживает ручьистого узора, то данные ямки не должны идентифицироваться как фасетка скола. Данный вид разрушения можно отнести к вязкому разрушению, инициированному путем зарождения, слияния и роста микропор. В свою очередь сплав $\mathrm{AMg} 4.5$ показал сильно выраженную рельефную смешанную структуру поверхности разрушения на которой присутствуют признаки вязкого ямочного разрушения и хрупкого скола.

С ростом скорости приложения нагрузки для сплава AMg3 (рис. $4, a, b$ ) до $\dot{\sigma}=4400 \mathrm{GPa} / \mathrm{s}$ деформационная картина становится более однородной с равномерным распределением одноразмерных ямок. Рентгеноструктурный анализ показывает равномерное распределение химических элементов из состава сплава по поверхности разрушения.

Поверхность разрушения сплава $\mathrm{AMg} 4.5$ при скорости $\dot{\sigma}=4400 \mathrm{GPa} / \mathrm{s}$ схожа со случаем квазистатического нагружения. Поверхность разрушения образована в основном путем скола и отрыва. Аналогичная картина наблюдается и при $\dot{\sigma}=5860 \mathrm{GPa} / \mathrm{s}$ (рис. 5). На поверхности разрушения образцов можно выделить отдельные области с глубокими ямками, которые являются инициаторами разрушения. Только в отличие от инициаторов на поверхности разлома у $\mathrm{AMg} 3$, в $\mathrm{AMg} 4.5$ разломы сопровождаются смесью хрупкого и вязкого 
разрушения (рис. 5, $c$ и $d$ соответственно). При этом в областях, соответствующих хрупкому разрушению, концентрируются элементы $\mathrm{Si}, \mathrm{Mn}$ и $\mathrm{Fe}$, в то время как, во-вторых, эти элементы отсутствуют. Поверхности разрушения сплава $\mathrm{AMg} 4.5$ содержат фасетки сколов (рис. $3, d, 4, d, 5, b)$. Наличие признаков хрупкого и вязкого разрушения на поверхности слома говорит о конкуренции двух механизмов разрушения - хрупкого и вязкого. Данный факт позволяет объяснить существенные различия в области деформирования образцов как в области квазистатических, так и в области динамических нагрузок.

\section{4. Заключение}

В работе получены диаграммы деформирования алюминиевых сплавов системы $\mathrm{Al}-\mathrm{Mg}$ в случае квазистатического нагружения и скоростные зависимости максимальных растягивающих напряжений в случае ударного воздействия. Показано, что с ростом концентрации $\mathrm{Mg}$ в сплаве увеличиваются пределы текучести и временной прочности при незначительном уменьшении пластичности. Однако эта закономерность нарушается при рассмотрении сплава $\mathrm{AMg} 4.5$ в литом состоянии без сформированной деформационной обработкой внутренней структуры.

Несмотря на пониженные пластические характеристики сплава $\mathrm{AMg} 4.5$ в области статического нагружения, данный материал демонстрирует динамическую прочность в терминах инкубационного времени на уровне AMg3, при равных значениях статической прочности. Расчеты с использованием структурно-временного подхода позволяют сделать вывод о достаточности уровня пластичности у сплава $\mathrm{AMg} 4.5$ для работы в реализованных скоростных режимах. Несмотря на существенные различия в деформационных процессах, происходящих в материалах $\mathrm{AMg} 4.5$ и $\mathrm{AMg} 3$, итоговые значения динамической прочности оказываются соизмеримыми, за тем исключением, что $\mathrm{AMg} 4.5$ является более хрупким и тем самым более чувствительным материалом к изменению внешнего воздействия. Это выражается в способности материала реагировать на ударное нагружение с более высокими скоростями роста напряжений и их максимальными значениями, превосходящими материал $\mathrm{AMg} 3$.

Выполненные исследования показали, что применение материала в экстремальных условиях нагружения требует всестороннего экспериментально-теоретического изучения его прочностных и пластических характеристик. Ухудшение свойств по одному из параметров может не оказать влияния на безопасную эксплуатацию конечного объекта в допустимом диапазоне ударных нагрузок.

\section{Финансирование работы}

Исследование выполнено при финансовой поддержке РФФИ (№ 19-31-60031).
Механические и структурные исследования проведены с использованием оборудования лаборатории СПбГУ „Механики перспективных массивных наноматериалов для инновационных инженерных приложений“, ресурсных центров Научного парка СПбГУ „Исследование экстремальных состояний материалов и конструкций“ и „Нанотехнологии“.

\section{Конфликт интересов}

Авторы заявляют, что у них нет конфликта интересов.

\section{Список литературы}

[1] J.R. Davis. Aluminum and aluminum alloys. Ohio: ASM International, Materials Park (1993). 783 p.

[2] G.V. Stepanov, V.V. Astanin, V.I. Romanchenko, A.P. Vashchenko, V.M. Tokarev, B.D. Chukhin, Y.P. Guk. Strength Mater. 15, 220 (1983).

[3] А.М. Брагов, А.К. Ломунов. ПМТФ 5, 168 (1988);

[4] A.M. Bragov, B.L. Karihaloo, Yu.V. Petrov, A.Yu. Konstantinov, D.A. Lamzin, A.K. Lomunov, I.V. Smirnov. J. Appl. Mech. Tech. Phys. 53, 6, 926 (2012).

[5] A.A. Gruzdkov, E.V. Sitnikova, N.F. Morozov, Y.V. Petrov. Mathem. Mech. Solid. 14, 72 (2009).

[6] A.A. Gruzdkov, S.I. Krivosheev, Y.V. Petrov. Phys. Solid State 45, 886 (2003).

[7] G.I. Kanel, S.V. Razorenov, A.A. Bogatch, A.V. Utkin, V.E. Fortov, D.E. Grady. J. Appl. Phys. 20, 467 (1997).

[8] Г.В. Гаркушин, Г.И. Канель, С.В. Разоренов. ФТТ 52, 2216 (2010).

[9] A.D. Evstifeev, I.V. Smirnov, Yu.V. Petrov. Phys. Solid State 61, 1062 (2019).

[10] A.D. Evstifeev, A.A. Chevrychkina, Y.V. Petrov. Mater. Phys. Mechan. 32, 258 (2017).

[11] W. Goldsmith, J.L. Sackman, C. Ewerts. Rock. Mech. Min. Sci. Geomech. 13, 303 (1976).

[12] S. Howe, W. Goldsmith, J. Sackman. Exp. Mech. 14, 337 (1974).

[13] Y.V. Petrov, I.V. Smirnov, A.A. Utkin. Mech. Solids 45, 476 (2010).

[14] Н.А. Златин, С.М. Мочалов, Г.С. Пугачев, А.М. Брагов. ФТТ 16, 1752 (1974).

[15] Я.Д. Вишняков, А.А. Бабарэко, С.А. Владимиров, И.В. Эгиз. Теория образования текстур в материалах и сплавах. Наука, М. (1979). 343 с.

[16] М.А. Штремель. Прочность сплавов. Ч. 2. МИСиС, M.(1997). $527 \mathrm{c}$.

[17] Y.V. Petrov, A.A. Utkin. Sov. Mater. Sci. 25, 153 (1989).

[18] Y.V. Petrov, N.F. Morozov. ASMEJ. Appl. Mech. 61, 710 (1994).

Редактор Т.Н. Василевская 